\title{
Percepción de los docentes y estudiantes hacia el proceso enseñanza-aprendizaje de la investigación en la Facultad de Ingeniería, Ciudad Universitaria, 2014
}

Jeileen Barahona Migueles ${ }^{1}$

Edwin Medina ${ }^{2}$

\section{RESUMEN}

La investigación científica es una herramienta fundamental para el desarrollo del ser, es el conocimiento de un pueblo, juega un papel fundamental en todos los niveles académicos; contexto en el cual nace esta investigación para conocer la percepción de los docentes y estudiantes sobre el proceso de enseñanza-aprendizaje de la investigación en la Facultad de Ingeniería de la Ciudad Universitaria, durante el tercer periodo académico de 2014.

Para realizar esta investigación se aplicó una encuesta dirigida a docentes que imparten clases asociadas a metodología y técnicas de investigación y alumnos que cursen la última clase relacionada con la investigación, según su plan de estudio.

Los datos fueron analizados, principalmente, mediante las técnicas de la estadística descriptiva. En tal sentido, se utilizaron distribuciones de frecuencias (absolutas y relativas) y medidas de tendencia central.

Como resultado se obtuvo información valiosa que permitirá determinar políticas adecuadas para mejorar los resultados en cuanto a la generación de investigación en la Facultad de Ingeniería. Cabe destacar que dentro de los principales hallazgos en dicha Facultad, se encontró que cuenta con profesores jóvenes y altamente calificados, con un rango de edades entre los 34 y 57 años, con cierta particularidad por el $100 \%$ de género masculino; un $89.9 \%$ estuvo muy de acuerdo en que le gusta

1 Beneficiaria de una beca de estudiante de posgrado de la Dirección de Investigación Científica y Posgrado, Maestría en Metodología de la Investigación Económica y Social, UNAH: miess@ unah.edu.hn

2 Profesor universitario y asesor, Dirección de Investigación Científica y Posgrado, UNAH: edwinhts @yahoo.com 
investigar y solo un $11.1 \%$ estuvo de acuerdo, por lo que se puede afirmar que existe una actitud positiva hacia la investigación por parte de los docentes de la Facultad de Ingeniería. Lo que coincide en una menor proporción con los resultados obtenidos por los estudiantes, así, la opción de acuerdo es la más elevada con $44.4 \%$, seguida de muy de acuerdo con $27.8 \%$, ni de acuerdo ni en desacuerdo con $16.7 \%$, mientras que las opciones de en desacuerdo y muy en desacuerdo presentaron porcentajes de $7.4 \%$ y $3.7 \%$, respectivamente.

Desde el punto de vista metodológico, esta investigación está promoviendo la aplicación de un método de investigación para generar conocimiento válido y confiable dentro del área de las ciencias sociales en general. En cuanto a su alcance, esta investigación abrirá nuevos caminos a investigadores que presenten situaciones similares a la que aquí se plantea, sirviendo como marco referencial a los mismos.

Palabras claves: enseñanza, aprendizaje, percepción, investigación, facultad de ingeniería universidad.

\section{ABSTRACT}

Scientific research is fundamental to the development of one's self, it is the knowledge of the people, and it plays a fundamental role in all academic levels, in this context, research begins by knowing the perception of teachers and students in regards to the process of research teaching \& learning at the Faculty of Engineering of the UNAH, during the third period of the 2014 academic year.

The research began with the application of a survey aimed at teachers who teach methodology courses and research techniques. It was also applied to students who have received research seminars in their last year's program.

The data was analyzed utilizing descriptive statistics. In this regard, frequency distributions (absolute and relative) and central tendency measures were used.

As a result, valuable information was obtained and it will identify appropriate policies to improve outcomes in terms of research generation in the Faculty of Engineering. As a result of the research, one fact stands out: the faculty has hired young and highly qualified teachers, with an age range between 34 and 57 years, mostly male teachers. 
Professors also show a positive attitude towards research, since $89.9 \%$ agreed to be in favor of developing research. In students, the results pointed out that only $44.4 \%$ were committed to research, $27.8 \%$ are neither in favor nor against and a smaller percentage does not agree at all.

From the methodological point of view, this research will help implement a better method for the generation of valid and reliable knowledge within the area of the social sciences in general. In terms of scope, this research opens new opportunities for researchers in similar situations.

Keywords: teaching, learning, perception, research, engineering faculty, university. 


\section{INTRODUCCIÓN}

Para Oseguera (2005) la capacidad que tienen las universidades hondureñas de producir conocimiento e innovación es limitada, pero existe una baja productividad de conocimiento, por no decir que es casi nula a nivel de pregrado, debido a que esta se da a nivel de posgrado y aun en este es limitada. Esto provoca que la información que se tiene de la realidad nacional y de las posibles soluciones a problemas reales sea reducida.

El presente estudio permitirá analizar si la baja producción de conocimiento a nivel de pregrado se ve limitada por la percepción que tienen los docentes y estudiantes hacia el proceso de enseñanza-aprendizaje de la investigación.

Para Hernández Pina (2002) la concepción que el profesor tenga del conocimiento, en nuestro caso sobre la investigación, afectará la forma en cómo el estudiante comprenda tal información. Es decir, la experiencia y la percepción que el docente tenga de un determinado fenómeno, influye en el desarrollo y comprensión del mismo. En tal sentido, teniendo en cuenta que en la Universidad la investigación es ejecutada en su mayoría por personas vinculadas a ella en calidad de docentes, los que son, al mismo tiempo, quienes desempeñan tareas de enseñanza y aprendizaje, la articulación de estas actividades en el ámbito institucional, grupal e individual debería ser casi natural.

La investigación científica no puede estar ausente de las tareas universitarias, mucho menos en países en vías de desarrollo, en donde algunas veces únicamente las universidades reúnen los recursos materiales y humanos indispensables para sustentar la actividad científica. En la universidad, la investigación debe contribuir no solo al descubrimiento de nuevas verdades, sino también a la transmisión de los métodos que permitan tales hallazgos, lo que precisamente garantiza la continuidad de la búsqueda. La universidad no debe limitarse a la simple transmisión de conocimiento, sino que debe empeñarse en su adelanto (Tunnermann, 2007). 


\section{METODOLOGÍA}

La metodología utilizada para el desarrollo del estudio es de tipo descriptivo, ya que su principal propósito es la caracterización de la percepción que tienen los docentes y estudiantes de la Facultad de Ingeniería acerca del proceso enseñanza-aprendizaje de la investigación.

La investigación se planteó desde un enfoque cuantitativo, pues se busca analizar la situación actual de la percepción de la enseñanza de la investigación a nivel de grado en la UNAH. El propósito del estudio es establecer una línea base sobre la temática, por tal razón se diseñó y aplicó un cuestionario mediante el cual se indagó sobre las siguientes dimensiones: metodología y estrategias de enseñanza-aprendizaje utilizadas por los docentes de clases relacionadas con la metodología de investigación, competencias y experiencia en investigación de los docentes, área de formación académica y capacitaciones recibidas en la temática, propósitos de la enseñanza de la investigación, propósitos de las asignaturas específicas, preferencias de enseñanza, textos y materiales utilizados, debilidades y áreas que se deben fortalecer y las percepciones globales de los docentes y estudiantes sobre el proceso de enseñanza-aprendizaje y la articulación de la enseñanza de la investigación en las asignaturas y espacios académicos diferentes.

Para la realización de este estudio se consideran dos tipos de informantes: los docentes que imparten clases relacionadas con la metodología de investigación y los estudiantes que recibieron, en el segundo periodo del año académico actual, las clases relacionadas con la metodología de la investigación de las cinco carreras de la Facultad de Ciencias Sociales. Se trabajó de la siguiente forma con los informantes del estudio:

1. Se tomó el universo de los docentes que imparten clases relacionadas con la metodología de investigación en el último trimestre del periodo académico, de las diferentes carreras de la Facultad de Ingeniería, a los que se aplicó una encuesta estructurada.

2. En el caso de los estudiantes se trabajó la totalidad de los matriculados en la clase Seminario de investigación en el tercer periodo de las diferentes carreras de la Facultad de Ingeniería, a quienes se les aplicó un cuestionario. Los datos del estudiante fueron obtenidos por los registros proporcionados por cada una de las carreras de la Facultad para su localización. 
En el caso de la percepción, el proceso metodológico estuvo constituido por un cuestionario dirigido a docentes y estudiantes, el cual consta de una serie de preguntas cerradas y de afirmaciones tipo escala de Likert, con cinco opciones de respuestas que van de 1 (muy en desacuerdo) a 5 (muy de acuerdo); con la misma se pretendió obtener información sobre la percepción que tienen sobre la enseñanza-aprendizaje de la investigación. Se entrevistó el total docentes que imparten actualmente las asignaturas de investigación o clases relacionadas con la misma.

Con los instrumentos recolectados mediante el cuestionario realizado al docente y estudiantes, se procedió a elaborar dos bases de datos en SPSS 20 (una de docentes y otra de estudiantes), en las cuales se digitaron todos los cuestionarios a partir de los cuales se llevó a cabo el análisis descriptivo de las variables (frecuencias y porcentajes). Todos los datos resultantes fueron evaluados a través de técnicas estadísticas y medidas de tendencia central apoyados en cuadros y gráficos que permiten identificar con claridad los resultados.

\section{SITUACIÓN}

En un intento por definir el concepto percepción se encuentra que la noción de este se deriva del término latino perceptio, que describe tanto a la acción como a la consecuencia (efecto) de percibir (es decir, de tener la capacidad para recibir mediante los sentidos las imágenes, impresiones o sensaciones externas 0 comprender y conocer algo). También puede interpretarse como la sensación interior que resulta de una impresión material hecha por los sentidos.

A la teoría que fundamenta la percepción se le denomina gestalt, que es un término alemán que significa forma. Esta teoría también conocida como la teoría de la forma, nace en Alemania bajo la autoría de Wertheimer, Koffka y Köhler, todos ellos constituidos como un movimiento durante las primeras décadas del siglo XX. Tales autores consideran la percepción como el proceso fundamental de la actividad mental y suponen que las demás actividades psicológicas como el aprendizaje, la memoria, el pensamiento, entre otros, dependen del adecuado funcionamiento del proceso de organización perceptual (Oviedo, 2004).

Existen diversas teorías sobre la percepción, algunas con una referencia permanente 
relacionada con las ciencias sociales y con la comunicación. Muchos autores asocian la percepción con la actitud, pero ambos términos tienen un significado distinto. Para Merleau-Ponty (2002), el mundo de la percepción es aquel que nos revelan nuestros sentidos y la vida que hacemos, a primera vista parece ser el mejor que conocemos, ya que no se necesitan ni instrumentos, ni cálculos para acceder a él.

En nuestro caso, una de las subvariables que nos permitirán medir la percepción del interés personal del docente es la actitud, apoyados en Aldana (2011). La actitud es el resultado de las tendencias positivas y negativas ante sucesos, acciones personales frente a los objetos, productos o servicios. Pérez (2009), apoyado por Vander (1987), define la actitud como la tendencia o predisposición adquirida y relativamente duradera para evaluar determinado modo de una persona, suceso 0 situación. Ander (1986) menciona que el término actitud designa un estado de disposición sicológica adquirida y organizada a través de la propia experiencia, que incita al individuo a reaccionar de una manera característica frente a determinadas personas, objetos y situaciones.

Se considera a la actitud como el resultado final del proceso de socialización, de cómo el sujeto va dando o construyendo las respuesta a las relaciones; la actitud permite medir la percepción, ya que denota la predisposición del individuo, ya sea esta favorable o desfavorable y en nuestro caso ante la enseñanza aprendizaje de la investigación en las aulas de clase; por lo tanto, la medición de este indicador es importante para la investigación, puesto que dará la referencia a las preferencias tanto de docentes como de estudiantes en lo relativo a la temática de interés (ver tabla 1).

Tabla 1. Variables consideradas en la investigación

\begin{tabular}{|l|l|l|}
\hline \multicolumn{2}{|c|}{ Percepción } \\
\hline Interés personal & $\begin{array}{l}\text { Vinculación entre ciencia, } \\
\text { sociedad y la investigación }\end{array}$ & $\begin{array}{c}\text { La investigación cientifica } \\
\text { como una forma de ser }\end{array}$ \\
\hline - Actitud & - Importancia & - Participación \\
- Motivación & - Relación con la carrera & - Habilidades \\
- Expectativas & - Didáctica & Compromiso \\
\hline
\end{tabular}

Fuente: elaboración propia. 


\section{Percepción en el proceso de enseñanza- aprendizaje: docentes}

\section{Datos generales}

El personal docente de la Facultad de Ingeniería fue el sujeto de estudio. Se observa que son profesores jóvenes y altamente calificados, con un rango de edades entre los 34 y 57 años, con cierta particularidad el $100 \%$ de género masculino, de los cuales el $66.7 \%$ proviene de la ciudad capital y el restante $33.3 \%$ del interior del país.

En cuanto a las clases relacionadas a la investigación, solo un docente ha impartido una asignatura adicional asociada al tema: Métodos de investigación I (la cual pertenece a otra facultad) y los ocho restantes únicamente cuentan con experiencia en el Seminario de investigación. Cabe mencionar que cada uno de ellos tiene carga académica asignada, pero con el enfoque de la carrera a la cual pertenecen.

\section{Interés personal}

1. Actitud: el personal docente encuestado en un $89.9 \%$ estuvo muy de acuerdo en que le gusta investigar y solo un $11.1 \%$ estuvo de acuerdo; a diferencia de las opciones muy en desacuerdo y en desacuerdo, las cuales no fueron consideras por los docentes, en vista que la actitud es un resultado de respuestas negativas o positivas hacia personas objetos 0 situaciones, según expertos en la materia como Vander y Ander. Por lo que se afirma que existe una actitud positiva hacia la investigación por parte de los docentes de la Facultad de Ingeniería. Al consultar si se consideraba un docente investigador, el $44.4 \%$ estuvo en desacuerdo, un $22.2 \%$ no estaba ni de acuerdo ni en desacuerdo, lo que llama la atención en vista de que una hubo un mayor grado de unanimidad en el gusto que tiene el docente por la investigación.

2. Motivación: el $77.8 \%$ está muy de acuerdo en que les interesa investigar, solo un $22.2 \%$ de los docentes está de acuerdo y un $37.5 \%$ manifiesta estar de acuerdo en que le gusta enseñar a investigar, aunque en la misma proporción consideran no estar de acuerdo ni en desacuerdo, por lo que existe una respuesta favorable a lo consultado, es decir, que hay un nivel de motivación no muy elevado por parte de los docentes hacia la enseñanza de la investigación.

3. Expectativa: es positiva, en vista que el $77.8 \%$ de los docentes de la Facultad de Ingeniería están muy de acuerdo en que les gustaría realizar investigaciones 0 
participar en proyectos de investigación en sus respectivas carreras, el restante personal está de acuerdo.

Vinculación entre la ciencia, sociedad y la investigación

Esta variable se evaluó utilizando indicadores como importancia, relación con la carrera, didáctica y conocimiento previo. Las consultas fueron las siguientes:

1. El enseñar a investigar en las universidades no es importante para la sociedad: el $100 \%$ considera estar muy en desacuerdo ante tal afirmación.

2. La investigación no contribuye a generar nuevos conocimientos: el $77.8 \%$ está muy en desacuerdo y un $11.1 \%$ en desacuerdo; sin embargo, el restante $11.1 \%$ está muy de acuerdo.

En resumen, se puede decir que el docente está consciente del nivel de importancia de que haya una estrecha relación entre la ciencia, la sociedad y la investigación. A su vez, el total de docente considera estar muy de acuerdo en que existe una vinculación entre la investigación y la carrera, por lo que es necesario que este tema sea tratado en todos los niveles de la Facultad.

La investigación científica como una forma de ser

Medida a través del compromiso, participación y habilidades: los estudiantes adquieren las competencias investigativas necesarias durante las clases de investigación; al respecto, un $33.3 \%$ informó estar de acuerdo con esta afirmación y en la misma proporción no están de acuerdo, ni en desacuerdo; lo cual indica que debe existir un plan de acción para medir el progreso del estudiante en temas investigativos y que el docente este consciente de ello (ver tabla 2). 


\section{Tabla 2. Percepciones de los docentes sobre el proceso enseñanza aprendizaje}

\section{Como docente}

Promedio

1. Me gusta la investigación cientifica

2. Enseñar a investigar requiere que me prepare más

3. Me interesa enseñar a investigar

4. Enseñar a investigar requiere disciplina

5. Me gustaría realizar investigaciones o participar en proyectos de investigación en mi carrera

6. El enseñar a investigar en las universidades no es importante para la sociedad

7. La investigación no contribuye a generar nuevos conocimientos

8. La investigación está vinculada con mi carrera

9. En el plan de estudios de mi carrera se aborda ampliamente la investigación

10. Enseñar a investigar implica utilizar textos adecuados

11. Tengo amplios conocimientos sobre metodologías de investigación

12. Me gusta más enseñar que investigar

13. La experiencia en investigación no es necesaria para impartir la asignatura

14. Mi compromiso solamente es dar a conocer la metodología de investigación

15. Tengo amplios conocimientos en programas estadísticos

16. Tengo la facilidad para investigar

17. Los estudiantes adquieren las competencias investigativas necesarias durante las clases de investigación

18. Logro que el aprendizaje de investigación sea integral

19. Me considero un docente-investigador

Fuente: elaboración propia. 


\section{Percepción en el proceso de enseñanza-aprendizaje: estudiantes}

\section{Datos generales}

Con relación a la información brindada por parte de los estudiantes de la Facultad de Ingeniería, se observa que la mayor proporción de estudiantes está representada por la Carrera de Ingeniería Eléctrica con un $52.7 \%$ del total de alumnos, seguidamente de la Carrera de Ingeniería en Sistemas con $27.3 \%$, Ingeniería Industrial $12.7 \%$, Ingeniería Civil $5.5 \%$ e Ingeniería Mecánica con un $1.8 \%$.

Se identificó, además, que el $78.2 \%$ de los estudiantes son hombres, mientras que el $21.8 \%$ son del sexo femenino. La edad promedio de los alumnos es de 24 años, con una edad máxima de 30 años y una edad mínima de 20 años. El estado civil de los estudiantes indica que la mayoría de esta población es soltera, dado que un $90.9 \%$ de estudiantes respondieron bajo esta categoría; mientras que un $5.5 \%$ se encuentran casados, un $1.8 \%$ informó viudez y un $1.8 \%$ se encuentra en la categoría: otra condición de estado civil, la cual incluye unión libre, madre soltera, padre soltero u otras distintas a las antes mencionadas.

Interés personal

Al inicio de este apartado se evaluó el interés de los estudiantes por la investigación científica, observándose que la opción de acuerdo es la más elevada con un $44.4 \%$, seguida de muy de acuerdo con $27.8 \%$, ni de acuerdo ni en desacuerdo con $16.7 \%$; mientras que las opciones en desacuerdo y muy en desacuerdo presentaron porcentajes de $7.4 \%$ y $3.7 \%$, respectivamente. Adicionalmente a ello, al observar el promedio de las preguntas asociadas a este apartado, se aprecia que existe una percepción positiva por parte de los estudiantes hacia la investigación.

Sobre la preparación para investigar de manera científica, el $59.3 \%$ estuvo muy de acuerdo en que aprender a investigar requiere de una mayor preparación por parte del estudiante, seguida de los que están de acuerdo con $33.3 \%$ y de los que no están ni de acuerdo ni en desacuerdo con $7.4 \%$; en promedio se obtuvo un resultado de 4.5 , es decir, que los alumnos están conscientes del nivel de compromiso que exige la investigación con un alto nivel de preparación. Al plantear que enseñar a investigar en las universidades no es importante para la sociedad, el 75,9 \% estuvo muy en desacuerdo; un 5,6\% de acuerdo y en igual porcentaje ni de acuerdo ni en desacuerdo; por otra parte, el $11,1 \%$ se mostró muy de acuerdo y el $1,9 \%$ en desacuerdo. 
Vinculación entre la ciencia, sociedad y la investigación

El $65,4 \%$ de los estudiantes indicaron estar muy de acuerdo en que les gustaría realizar investigaciones o participar en proyectos de investigación en su carrera; un $17 \%$ se mostró de acuerdo, el $15,4 \%$ ni de acuerdo ni en desacuerdo, dejando un $1,9 \%$ a los estudiantes que dijeron estar muy en desacuerdo.

El 33,3\% de los estudiantes consideran que en el plan de estudios de su carrera se aborda ampliamente la investigación, un $22,2 \%$ ni de acuerdo ni en desacuerdo, el $20,4 \%$ muy en desacuerdo, $16,7 \%$ de acuerdo y un $7,4 \%$ muy de acuerdo.

La investigación científica como una forma de ser

El 35,2 \% está muy en desacuerdo en que la experiencia en investigación no es necesaria para trabajar; un 27,8 \% ni de acuerdo ni en desacuerdo; el $18,5 \%$ en desacuerdo; $9,3 \%$ de acuerdo y el mismo porcentaje muy de acuerdo. Con esto se confirma que existe un compromiso por ser mejores, al determinar que sí se requiere de investigación en todos los ámbitos.

Al plantear a los estudiantes que su compromiso es conocer ligeramente la metodología de investigación, el 35,2 \% afirmó no estar ni de acuerdo ni en desacuerdo; el 24,1 $\%$ de acuerdo; el $16,7 \%$ muy en desacuerdo; un $14,8 \%$ muy de acuerdo y el restante $9,3 \%$ en desacuerdo (ver tabla 3 ). 
Tabla 3. Percepciones de los estudiantes sobre el proceso enseñanza-aprendizaje

\begin{tabular}{|c|c|}
\hline Como estudiante & Promedio \\
\hline 1. Me gusta la investigación científica & 3.9 \\
\hline 2. Aprender a investigar requiere que me prepare más & 4.4 \\
\hline 3. Me interesa aprender a investigar & 4.3 \\
\hline 4. Aprender a investigar requiere disciplina & 4.5 \\
\hline $\begin{array}{l}\text { 5. Me gustaría realizar investigaciones o participar en proyectos de investigación } \\
\text { en mi carrera }\end{array}$ & 4.4 \\
\hline 6. El enseñar a investigar en las universidades no es importante para la sociedad & 1.7 \\
\hline 7. La investigación no contribuye a generar nuevos conocimientos & 1.6 \\
\hline 8. La investigación está vinculada con mi carrera & 4.4 \\
\hline 9. En el plan de estudios de mi carrera se aborda ampliamente la investigación & 2.6 \\
\hline 10. Aprender a investigar implica utilizar textos adecuados & 4.1 \\
\hline 11. Tengo amplios conocimientos sobre metodologías de investigación & 2.8 \\
\hline 12. Me gusta más estudiar los temas de mi carrera que investigar & 3.2 \\
\hline 13. La experiencia en investigación no es necesaria para trabajar & 2.4 \\
\hline 14. Mi compromiso es conocer ligeramente la metodología de investigación & 3.1 \\
\hline 15. Tengo amplios conocimientos en programas estadísticos & 2.9 \\
\hline 16. Tengo la facilidad para investigar & 3.3 \\
\hline $\begin{array}{l}\text { 17. Los estudiantes adquirimos las competencias investigativas necesarias } \\
\text { durante las clases de investigación }\end{array}$ & 3.2 \\
\hline $\begin{array}{l}\text { 18. En las clases de investigación se logra que el aprendizaje de investigación } \\
\text { sea integral }\end{array}$ & 3.5 \\
\hline 19. Me considero un investigador & 2.6 \\
\hline
\end{tabular}

Fuente: elaboración propia. 


\section{CONCLUSIONES}

1. La percepción de los docentes y estudiantes hacia el proceso de enseñanza -aprendizaje de la investigación en la Facultad de Ingeniería de la Ciudad Universitaria, durante el último trimestre del año 2014, es positiva, ya que los docentes están de acuerdo y muy de acuerdo con el proceso de enseñanza de la investigación; en tanto que los estudiantes consideran estar en un $44.4 \%$ de acuerdo; seguido de muy de acuerdo con un $27,8 \%$, ni de acuerdo ni en desacuerdo con $16,7 \%$; mientras que las opciones en desacuerdo y muy en desacuerdo presentaron porcentajes de $7,4 \%$ y $3,7 \%$, respectivamente.

2. Se puede concluir que tanto para docentes como estudiantes de la Facultad de Ingeniería demuestran una actitud positiva hacia la investigación, pero en varios aspectos tanto alumnos como docentes presentan debilidades; por ejemplo, en la capacidad del estudiante para identificar la fundamentación teórica. El estudiante pese a la formación no se considera un investigador, por eso es necesario ir generando políticas de concienciación al alumno.

3. Se cuenta con personal altamente calificado en la Facultad de Ingeniería, con un nivel académico en grado de licenciatura, pero con altos conocimientos en investigación, los que pueden ser utilizados para generar mayor investigación científica en su área de trabajo. Dado que los alumnos muestran interés por la investigación, se puede decir que ambas partes, docentes y alumnos, pueden fortalecer las investigaciones realizadas en cada carrera.

\section{BIBLIOGRAFÍA}

Alvarado, M. y Flores, F. (2009). Percepciones y supuestos sobre la enseñanza de la ciencia: Las concepciones de los investigadores universitarios. Revista Universitaria UNAM.

Aldana de Becerra, Gloria. (2008). Enseñanza de la investigación y epistemología de Ios docentes. Colombia: Universidad de La Sabana, Cundinamarca.

Aldana, G. y Joya, N. (2011). Actitudes hacia la investigación científica en docentes de metodologías de investigación. Colombia: Tabula Rasa.

Bain, Ken. (2007). Lo que hacen los mejores profesores universitarios. Universidad de Valencia: PUV. 
Barrón Ruíz, A. (1993). Aprendizaje por descubrimiento: principios y obligaciones inadecuadas. Investigación y experiencias didácticas, 11(1), 3-11.

Dómenech, F. y otros. (2004). Percepción del proceso de enseñanza/aprendizaje desarrollado en Psicoestadística I y su incidencia en el rendimiento. Psicothema, 16(1), 32-38.

Dómench, F. (1990). Proceso de enseñanza/aprendizaje universitario: aspectos teóricos y prácticos. España: Universitat Haumel I.

Hernández Pina, Fuensanta. (2002). Docencia e investigación en educación superior. Facultad de Educación, Universidad de Murcia.

Jerome, Bruner. (2010). Dos teorías cognitivas, dos formas de significar, dos enfoques para la enseñanza de la ciencia. Psicogente, 13(24), 329-346.

Marcelo, Carlos. (2009). Marcos generales de investigación sobre la enseñanza en la universidad. España: Universidad de Sevilla.

Oviedo, G. L. (2004). La definición del concepto de percepción en psicología con base en la teoría gestalt. Revista de Estudios Sociales, 89-96.

Pérez, Carlos. (2009). Medición de la percepción del consumidor en una empresa de control de plagas, aplicando el modelo de Likert y los modelos de atención al cliente. Centro de Investigaciones Económicas, Administrativas y Sociales.

Rama, C. (2007). Los posgrados en América Latina y el Caribe en la sociedad del conocimiento. México: UDUAL.

Rojas, M. y Méndez, R. (2013). Cómo enseñar a investigar. Un reto para la pedagogía universitaria. Colombia: Universidad Industrial de Santander. 